\title{
Observation of Tritium Distribution in $\mathrm{V}-4 \mathrm{Cr}-4 \mathrm{Ti}$ alloy by Tritium Radioluminography*1
}

\author{
Hirofumi Homma ${ }^{1, * 2}$, Hideyuki Saitoh ${ }^{1}$, Toshihei Misawa ${ }^{1}$ and Toshiyuki Ohnishi ${ }^{2}$ \\ ${ }^{1}$ Department of Materials Science and Engineering, Muroran Institute of Technology, Muroran 050-8585, Japan \\ ${ }^{2}$ Central Institute of Radioisotope Science, Hokkaido University, Sapporo 060-0815, Japan
}

\begin{abstract}
Tritium distribution in $\mathrm{V}-4$ mass $\% \mathrm{Cr}-4$ mass $\% \mathrm{Ti}$ alloy has been observed by the tritium radioluminography, and the relation between the tritium distribution and the constituent elements of the alloy have been investigated. In the as-cast specimen, tritium concentration has been higher in the titanium enriched region, that is, the local tritium concentrations at the lower and higher titanium region are 156 and 202 mol $\mathrm{ppb}$, respectively. The tritium distribution has shown a strong correlation with the titanium segregation in the interdendritic region. In the heat treated specimen, the tritium distribution has been rarely affected by the interdendritic titanium segregation. Tritium concentration at the central area and at the surrounding area in the specimen surface have been 209 and 147 mol ppb, respectively. It has been thought that this accumulation of tritium to the central area is caused by the Gorsky effect which generated due to the constraint condition of the stress field in the plate shaped specimen. In the heat treated specimen, the tritium distribution has been more strongly affected by the shape of the specimen than by the titanium segregation in it.
\end{abstract}

(Received October 25, 2001; Accepted January 7, 2002)

Keywords: tritium, Vanadium-4 mass\%Chromium-4 mass\%Titanium alloy, imaging plate, tritium radioluminography

\section{Introduction}

Tritium is the radioactive isotope of hydrogen, and emits only low energy $\beta$-rays. Thus, tritium has been used to observe hydrogen distribution in metals. Tritium radioluminography ${ }^{1)}$ is a useful technique to visualize hydrogen distribution in materials quantitatively, based on the photo-stimulated luminescence of the imaging plate (IP).$^{2-4)}$ In this method, tritium charged specimen is placed on the IP, and then the twodimensional distribution and intensity of the $\beta$-rays emitted from tritium are detected. The quantitativeness of the radioluminography for the tritium distribution is excellent because the IP possesses an extremely high sensitivity and wide dynamic range of about five orders for the $\beta$-rays. ${ }^{5)}$ By the tritium radioluminography, it is possible to observe not only the two-dimensional hydrogen distribution but also the hydrogen concentration at the local area on the specimen surface.

In our previous work, we performed the quantitative observation of the hydrogen distribution in pure vanadium, 6,7 ) some vanadium alloys ${ }^{8-13)}$ and $\mathrm{Ti}-\mathrm{Cr}$ alloy ${ }^{14)}$ by the tritium radioluminography, and showed the effect of the microstructure or constituent element on the tritium distribution in these materials. In the present work, tritium radioluminography is applied to $\mathrm{V}-4$ mass $\% \mathrm{Cr}-4$ mass $\% \mathrm{Ti}$ (mass\% will be omitted hereafter) alloy which is one of the candidate for the first wall materials of the nuclear fusion reactor. ${ }^{15)}$ The relation between the tritium distribution and both the constituent elements and microstructure is investigated.

\section{Experimental Procedure}

$\mathrm{V}-4 \mathrm{Cr}-4 \mathrm{Ti}$ alloy was prepared by arc-melting vanadium $(99.95 \%)$, chromium (99.9\%) and titanium (99.9\%) in high

\footnotetext{
${ }^{* 1}$ This Paper was Presented at the Autumn Meeting of the Japan Institute of Metals, held in Fukuoka, on September 24, 2001.

${ }^{* 2}$ Graduate Student, Muroran Institute of Technology.
}

purity argon atmosphere. The button-like ingot was cut to make plate specimens $1 \mathrm{~mm}$ in thickness. Some specimens were sealed in quartz tube after evacuating by a rotary pump and diffusion pump, and were heat treated at $1473 \mathrm{~K}$ for $24 \mathrm{~h}$ and then quenched into water. The plate specimens were polished using abrasive paper and mirror finished using alumina paste with $0.05 \mu \mathrm{m}$ particles.

Tritium addition into the specimens was performed by an electrochemical cathodic charging method at room temperature. The electrolyte was $0.1 \mathrm{kmol} / \mathrm{m}^{3} \mathrm{NaOH}$ aqueous solution containing tritium of $1.9 \mathrm{PBq} / \mathrm{m}^{3}$. The current density and charging period were $200 \mathrm{~A} / \mathrm{m}^{2}$ and $2 \mathrm{~h}$, respectively. The ratio of tritium atom $N_{\mathrm{T}}$ to protium atom $N_{\mathrm{H}}$ in the electrolyte was $N_{\mathrm{T}} / N_{\mathrm{H}}=1.5 \times 10^{-5}$. Then the tritium added specimens were immersed into dilute acetic acid, washed with water, rinsed in ethyl alcohol and then dried. The alkaline electrolyte adsorbed on the specimen surface is eliminated by this treatment.

The tritium added specimen was placed on an IP, Fujix TR2040, for $24 \mathrm{~h}$ to expose it to the $\beta$-rays emitted from tritium in the specimen. In this treatment, a tritium-labelled microscale (Amersham RPA510) which is a plastic film containing eight levels of known amount of tritium was placed on the IP to translate the tritium radioactivity into the tritium concentration. The detail of the qualitative analysis of tritium concentration is described in the previous papers. ${ }^{1,7)}$ After the exposure, two-dimensional mapping of the intensity of $\beta$-rays recorded in the IP was measured by means of an IP-reader (Fujix FDL5000) and the tritium radioluminograph was obtained. The pixel size of the radioluminograph obtained by the IP-reader was $25 \mu \mathrm{m} \times 25 \mu \mathrm{m}$, meaning that the resolution of the radioluminograph is $25 \mu \mathrm{m}$.

After the radioluminography, tritium in the specimen was exhausted by heating at $473 \mathrm{~K}$ for $1 \mathrm{~h}$ in vacuum and then the distribution of constituent elements and microstructures were examined. The distribution of the constituent elements 
of the specimen such as vanadium, chromium and titanium was observed by means of the wavelength dispersive X-ray spectrometer (WDX, JEOL JXA-8900). The crystal structure of the specimens before and after hydrogen charging was examined by means of a X-ray diffractometer using $\mathrm{Cu}-\mathrm{K} \alpha$ radiation.

\section{Results and Discussion}

Figure 1 shows X-ray diffraction profiles of the $\mathrm{V}-4 \mathrm{Cr}-4 \mathrm{Ti}$ specimen, (a) and (b) being as-cast and heat treated specimen, respectively, before and after hydrogen charging. It is confirmed that these specimens have BCC single phase before and after hydrogen charging. The 110 peak positions of both the as-cast specimen and heat treated specimen are slightly different for the hydrogen uncharged specimens, that is, the 110 peak position shifts to higher diffraction angle by the heat treatment. The calculated lattice spacings of both specimen shows that the (110) lattice spacings decrease $0.18 \%$ after the heat treatment. This shows that the lattice strain introduced during arc-melting is modified by the heat treatment.

For the hydrogen charged specimens, the peak positions of both the as-cast specimen and heat treated specimens shift to lower diffraction angle, indicating that the lattice expansion occurs due to the hydrogen dissolution. Figure 2 shows the change of the lattice spacings of both the as-cast specimen and heat treated specimens after hydrogen charging. The change of the lattice spacings in the heat treated specimen is larger
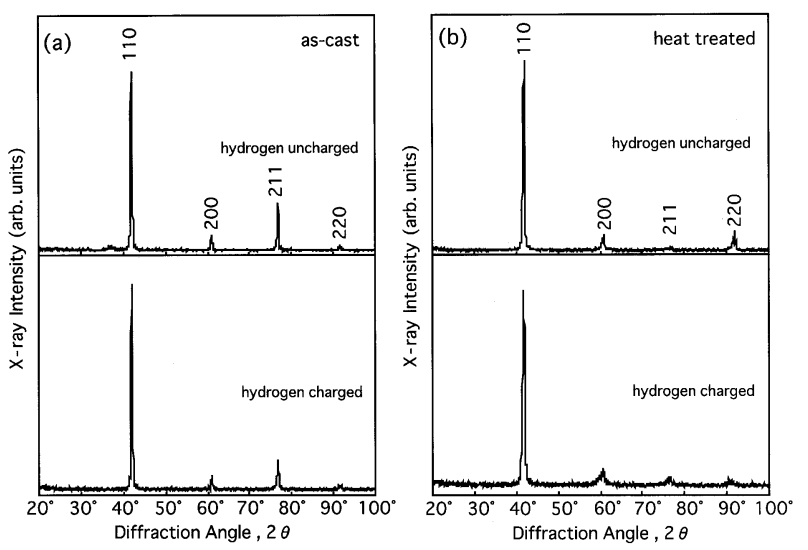

Fig. 1 X-ray diffraction profiles of (a) as-cast and (b) heat treated $\mathrm{V}-4 \mathrm{Cr}-4 \mathrm{Ti}$ specimens before and after hydrogen charging.

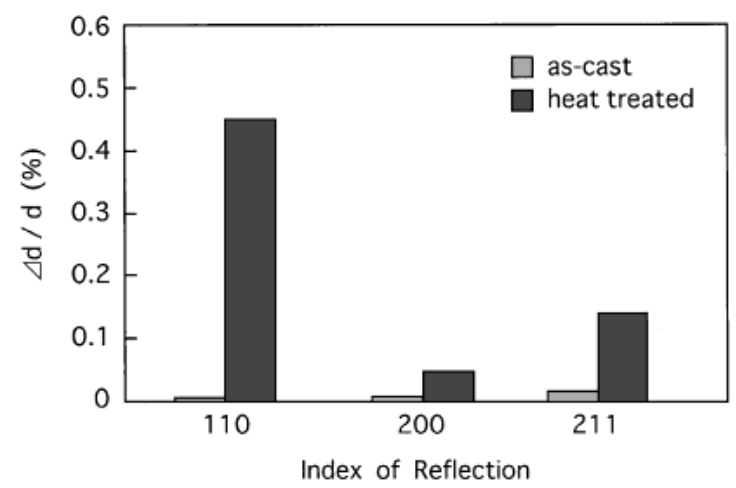

Fig. 2 Change of the lattice spacings in as-cast and heat treated $\mathrm{V}-4 \mathrm{Cr}-4 \mathrm{Ti}$ specimens by the hydrogen charging. than that in the as-cast specimen. This shows that the amount of hydrogen dissolved into the heat treated specimen is larger than that in the as-cast specimen.

Figure 3(a) shows a tritium mapping image of the as-cast $\mathrm{V}-4 \mathrm{Cr}-4 \mathrm{Ti}$ specimen on tritium added surface obtained by the tritium radioluminography. Tritium is observed to be nonuniformly distributed. The relative concentration of the tritium is displayed graphically by a calor scale bar in sixteen steps below Fig. 3(a), that is, the regions of higher, middle and lower hydrogen concentration in the radioluminograph is indicated by the colors of red, yellow and blue, respectively. Tritium concentration seems to vary depending on the microstructure in the specimen. Figure 3(b) to (d) show the mapping images of vanadium, chromium and titanium, respectively, obtained by WDX. Figure 3(e) to (h) show the enlarged pictures of the tritium, vanadium, chromium and titanium, respectively, in the area of square rounded portion in Fig. 3(a). In these mapping images, titanium is clearly observed to be segregated in the interdendritic region, while chromium has a weak tendency for segregation. It seems that both the vanadium and chromium are complementally distributed to the titanium distribution. By comparing the distribution of tritium and titanium, it is found that the tritium concentration at the microscopic region correlates closely with the titanium distribution. The local tritium concentrations at the lower titanium region (1) and higher titanium region (2) in Fig. 3(e) are 156 and $202 \mathrm{~mol} \mathrm{ppb}$, respectively. By considering that the heat of hydride formation is higher in titanium and lower in vanadium, ${ }^{16)}$ hydrogen is thought to be easily accumulated to the titanium rich region. And it seems that the lattice strain between the matrix and the titanium segregation caused by the difference of atomic radius between titanium and chromium also accumulate the hydrogen. Therefore, it is concluded that the tritium distribution is strongly affected by the titanium segregation in the interdendritic region.

Figure 4(a) shows a mapping image of the heat treated V$4 \mathrm{Cr}-4 \mathrm{Ti}$ specimen on the tritium added surface, and Fig. 4(b) to (d) show the mapping images of vanadium, chromium and titanium, respectively. Figure 4(e) to (h) show the enlarged pictures of the tritium, vanadium, chromium and titanium, respectively, in the area of square rounded portion in Fig. 4(a). In Fig. 4(a), tritium is observed to be accumulated to the central area in the specimen, and the tritium concentration is relatively low at the surrounding area. The tritium concentrations at the region (1) and (2) in Fig. 4(a) are 147 and $209 \mathrm{~mol} \mathrm{ppb}$, respectively. This higher tritium concentration in the central area is not attributed to the deformation of specimen but the accumulation of hydrogen to this area, because the difference of thickness between the central area and surrounding area after the tritium addition was less than $0.1 \mathrm{~mm}$ and the maximum range of $\beta$-rays emitted from tritium is about $3 \mathrm{~mm}$ in air. The accumulation of tritium to the central area was observed in the plate specimens of palladium $^{9)}$ and $\mathrm{V}-50 \mathrm{~mol} \% \mathrm{Ti}^{\text {alloy }}{ }^{10)}$ in our previous paper. It is reported ${ }^{17)}$ that the thickness increases as the hydrogenation progress while the length and width decreases due to the influence of constraint condition of the stress field in the palladium plate specimen. If we assume that the specimen is plane strain state, the deformation by the hydrogen penetration to the thickness direction is easily generated in the cen- 

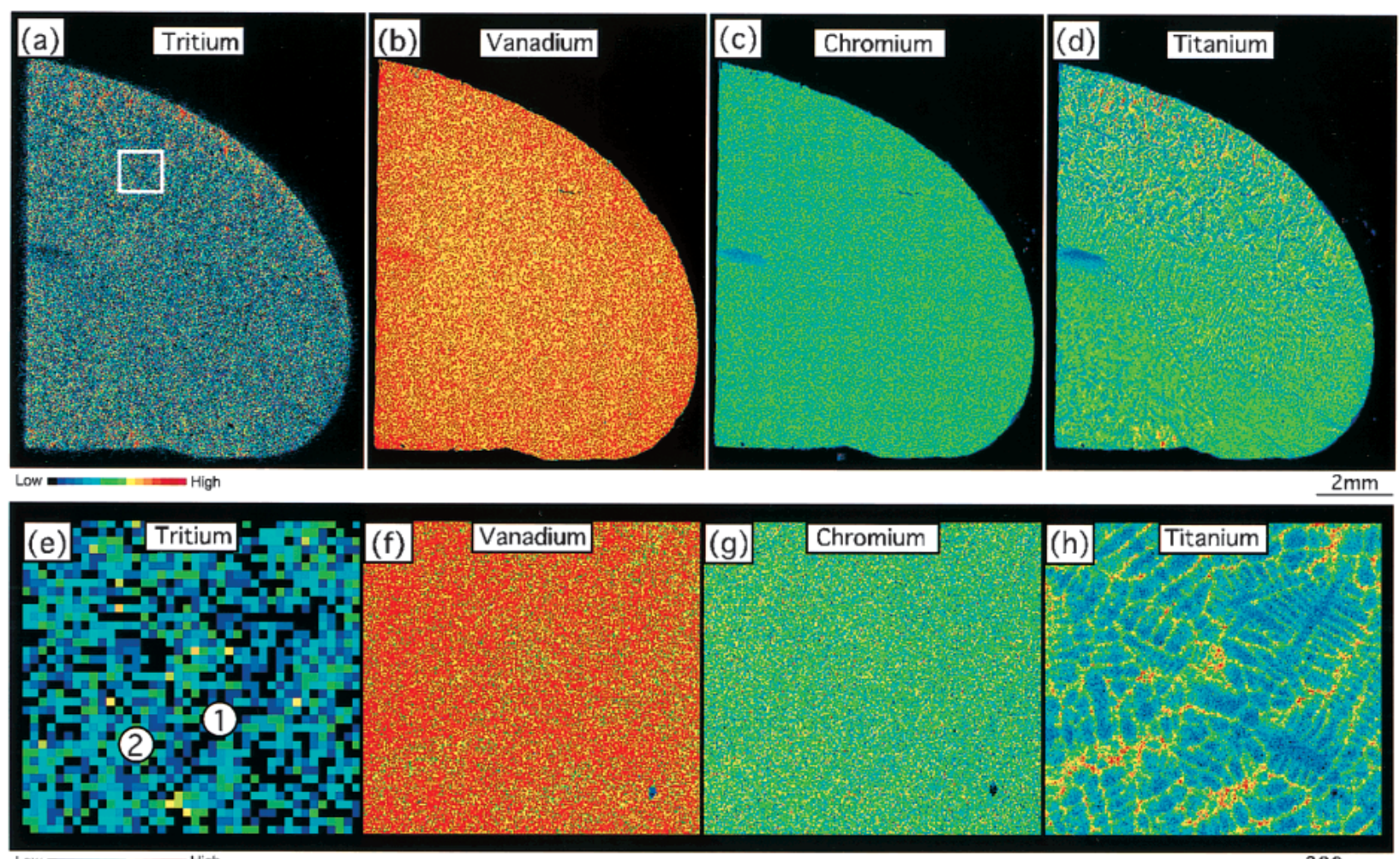

ow $=$ High

$300 \mu \mathrm{m}$

Fig. 3 (a) Mapping image of tritium on the tritium added surface of as-cast $\mathrm{V}-4 \mathrm{Cr}-4 \mathrm{Ti}$ specimen obtained by tritium radioluminography, (b) to (d) the mapping images of vanadium, chromium and titanium, respectively, obtained by WDX. (e) to (h) the enlarged pictures of tritium, vanadium, chromium and titanium, respectively, in the area of square rounded portion in (a).
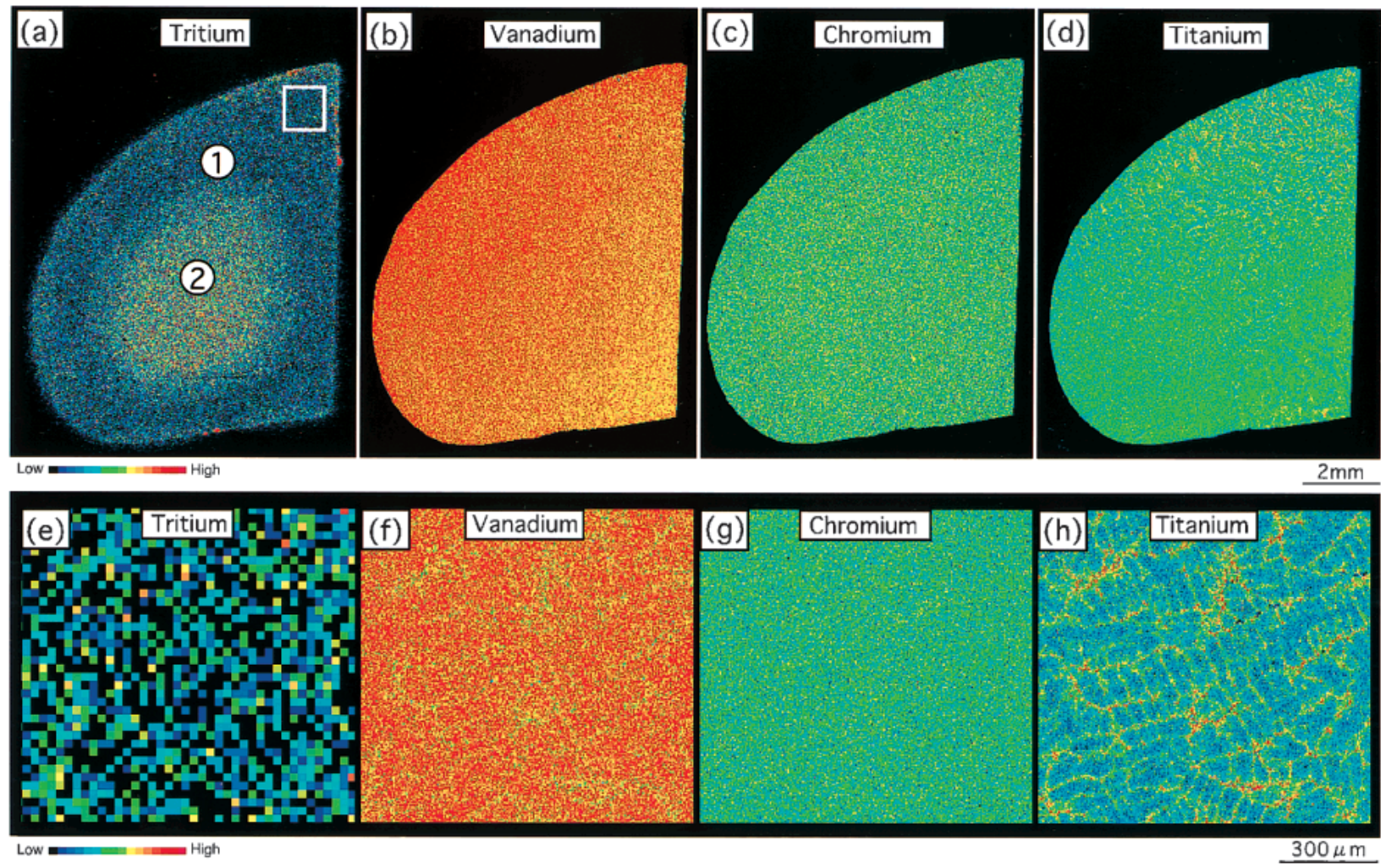

Fig. 4 (a) Mapping image of tritium on the tritium added surface of heat treated $\mathrm{V}-4 \mathrm{Cr}-4 \mathrm{Ti}$ specimen obtained by tritium radioluminography, (b) to (d) the mapping images of vanadium, chromium and titanium, respectively, obtained by WDX. (e) to (h) the enlarged pictures of tritium, vanadium, chromium and titanium, respectively, in the area of square rounded portion in (a). 
tral area than in the surrounding area because of the constraint condition of the stress field in the plate shaped specimen. It is thought that this stress field results in the accumulation of hydrogen to the central area by the Gorsky effect ${ }^{18)}$ which moderates the stress in the plate shaped specimen.

At the microscopic area, Fig. 4(e), tritium seems to be randomly distributed, that is, tritium distribution is rarely affected by the microstructure in contrast to the as-cast specimen. In Fig. 4(h), titanium is observed to be segregated in the interdendritic region similar to the as-cast specimen in Fig. 3 (h), and both the vanadium and chromium are also observed to be complementally distributed to the titanium distribution. Comparing the tritium and titanium distribution, it is found that the tritium distribution has a weak tendency to correlate with the titanium segregation in the interdendritic region. The interface between the titanium rich interdendritic region and titanium poor dendritic region becomes obscure compared with that in as-cast specimen. This loss of the sharpness of the interface is due to the diffusion of segregated titanium into the matrix by the heat treatment. This heat treatment also cause the modification of the lattice strain introduced during arc-melting, as mentioned in the result of X-ray diffraction experiment in Fig. 1. It is thought that the shaded interface of the dendritic structure and the decrease of the remaining strain causes the reduction of accumulation power of tritium in the heat treated specimen. Therefore, it is concluded that tritium distribution is more strongly affected by the shape of the specimen than by the titanium segregation in it.

\section{Conclusions}

The distribution of tritium and constituent elements in $\mathrm{V}-$ $4 \mathrm{Cr}-4 \mathrm{Ti}$ alloy was observed by the tritium radioluminography and by the wavelength dispersive X-ray spectrometry, respectively, and the relation between the distribution of them was examined. In the as-cast specimen, the tritium distribution strongly correlates with the titanium segregation in the interdendritic region. While, in the heat treated specimen, tritium accumulates to the central area in the specimen, and tritium distribution is rarely affected by the titanium segregation. The tritium distribution is strongly affected by the constraint condition of the stress field generated by the hydrogen penetration in the plate shaped specimen.

\section{Acknowledgements}

This work was supported by a Grant-in-Aid for Scientific Research (B) from the Ministry of Education, Culture, Sports, Science and Technology of Japan.

\section{REFERENCES}

1) H.Saitoh: JIM seminar text, "Saishin no Suiso no Kensyutuhou to Suisozeika bousihou”, Japan Inst. Metals (1999) pp. 33-39.

2) Y. Amemiya: J. Synchrotron Radiat. 2 (1995) 13-21.

3) H. Ayato, N. Mori, J. Miyahara and T. Oikawa: J. Electron Microsc. 39 (1990) 444-448.

4) M. Sonoda, M. Takano, J. Miyahara and H. Kato: Radiology 148 (1983) 833-838.

5) BAS Technical Information No. 2, Bio-Imaging Analyzer Group, Fuji Photo Film Co., LTD.

6) H. Saitoh, T. Hishi, T. Misawa, T. Ohnishi, Y. Noya, T. Matuzaki and T. Watanabe: J. Nucle. Mater. 258-263 (1998) 1404-1408.

7) H. Saitoh, T. Misawa, Y. Noya and T. Ohnishi: Mater. Trans., JIM 40 (1999) 692-695.

8) H. Saitoh, H. Naganuma, T. Misawa, T. Tamura, T. Kuriiwa, A. Kamegawa, M. Okada and T. Ohnishi: J. Japan Inst. Metals 63 (1999) 1085-1088.

9) H. Saitoh and T. Ohnishi: J. Mater. Sci. Lett. 19 (2000) 751-753.

10) H. Homma, H. Saitoh, T. Misawa and T. Ohnishi: J. Japan Inst. Metals 64 (2000) 295-298.

11) H. Saitoh, H. Homma, T. Misawa, T. Tamura, T. Kuriiwa, A. Kamegawa, M. Okada and T. Ohnishi: J. Japan Inst. Metals 64 (2000) 763-766.

12) H. Saitoh, H. Homma, T. Misawa and T. Ohnishi: Mater. Trans. 42 (2001) 399-402.

13) H. Homma, H. Saitoh, T. Misawa and T. Ohnishi: J. Japan Inst. Metals 65 (2001) 163-166.

14) H. Homma, H. Saitoh, T. Misawa and T. Ohnishi: J. Japan Inst. Metals 65 (2001) 807-810.

15) H. Matsui, K. Fukumoto, D. L. Smith, H. M. Chung, W. van Witzenburg and S. N. Votinov: J. Nucle. Mater. 233-237 (1996) 92-99.

16) Y. Osumi: Suiso kyuzou Goukin, (Agune Co. Ltd., 1993) pp. 73.

17) Y. Jung and Y. Sakai: J. Soc. Mat. Sci., Japan 50 (2001) 999-1006.

18) Hydrogen in Metals I Ed. By G. Alefeld and J.Völkl (Springer-Verlag, 1978) pp. 62. 